\title{
Pre-B cell leukemia transcription factor 3 induces inflammatory responses in human umbilical vein endothelial cells and murine sepsis via acting a competing endogenous RNA for high mobility group box 1 protein
}

\author{
YUNZHONG ZHANG $^{1 *}$, JING FENG $^{2 *}$, JIZHEN CUI $^{1}$, GUOZHENG YANG $^{1}$ and XIANAI ZHU ${ }^{1}$ \\ ${ }^{1}$ Intensive Care Unit; ${ }^{2}$ Department of Gynecology, Qingzhou Hospital, \\ Taishan Medical University, Qingzhou, Shandong 262500, P.R. China
}

Received March 30, 2017; Accepted September 8, 2017

DOI: $10.3892 / \mathrm{mmr} .2018 .8609$

\begin{abstract}
The present study investigated the roles of pre-B cell leukemia transcription factor 3 (PBX3) in sepsis. Reverse transcription-quantitative polymerase chain reaction and western blot analysis indicated that overexpression of the PBX 3'-untranslated region (UTR) promoted high mobility group box 1 (HMGB1) protein expression in human umbilical vein endothelial cells (HUVECs) $(\mathrm{P}<0.01)$. Furthermore, post-treatment of PBX3 small interfering (si)RNA suppressed lipopolysaccharide (LPS)-mediated HMGB1 release and attenuated HMGB1-mediated hyperpermeability and leukocyte migration in HUVECs and septic mice $(\mathrm{P}<0.01)$. Additionally, post-injection of PBX3 siRNA also induced the downregulation of cecal ligation and puncture-induced HMGB1 release, production of IL-6 and mortality $(\mathrm{P}<0.01)$. Mechanistically, the 3'UTRs of PBX3 and HMGB1 were identified to harbor six common micro (mi)RNA binding sites, and PBX 3'UTR increased HMGB1 expression in a 3'UTR- and miRNA-dependent manner. Notably, the coding sequence of PBX3 did not increase HMGB1 expression in HUVECs. Collectively, the present study indicates that PBX 3'UTR may induce inflammatory responses and sepsis via acting as a competing endogenous RNA for HMGB1.
\end{abstract}

\section{Introduction}

Despite improvements in sepsis treatment, morbidity and mortality rates from severe sepsis remain high, and current

Correspondence to: Dr Yunzhong Zhang, Intensive Care Unit, Qingzhou Hospital, Taishan Medical University, 1726 Linglong Mountain Road, Qingzhou, Shandong 262500, P.R. China

E-mail: yunzhong_zhang@163.com

*Contributed equally

Key words: high mobility group box 1, inflammation, lipopolysaccharide, pre-B cell leukemia transcription factor 3, sepsis therapeutic choices are limited (1). Therefore, it is critical to uncover the mechanisms mediating sepsis progression.

The competing endogenous (ce)RNA theory has been proposed to mediate tumor progression, which suggests that RNA transcripts, including mRNA, long non-coding RNA, circular RNA and pseudogenes, may modulate each other's expression by competing for common micro RNA (miRNA or miR) binding sites (2). Physiological roles of ceRNA have been revealed in various types of cancer, including breast cancer (3), lung cancer (4), hepatocellular carcinoma (5) and glioma (6). However, the roles of ceRNA in sepsis remain unknown.

High mobility group box 1 (HMGB1), which has been studied for its intracellular role as a DNA binding protein facilitating gene transcription, is a pro-inflammatory cytokine that has been studied for over a decade $(7,8)$. HMGB1 has been identified as a critical mediator of sepsis as it is released from activated macrophages (9). A recent study has revealed that pre-B cell leukemia transcription factor 3 (PBX3) promotes gastric cancer invasion and metastasis by inducing epithelial-mesenchymal transition (10). PBX3 is a putative biomarker of aggressive prostate cancer (11) and promotes the migration and invasion of colorectal cancer cells via activating the mitogen-activated protein kinase/extracellular signal-regulated kinase signaling pathway (12). Furthermore, previous results have demonstrated that with a greater number of shared miRNAs, there is an increased probability of existing in a ceRNA network (13). Notably, the PBX3- and HMGB1-3'untranslated regions (UTRs) have been demonstrated to share six miRNA binding sites by bioinformatics methods (such as starbase.sysu.edu.cn/index.php and www.microrna.org/microrna/getExprForm.do) (14). Therefore, the present study investigated whether PBX3 may act as a ceRNA for HMGB1 in regulating sepsis.

In the present study, reverse transcription-quantitative polymerase chain reaction (RT-qPCR) and western blot analysis were used to investigate the expression of PBX3 3'UTR and HMGB1. Additionally, the effects of post-treatment with PBX3 small interfering (si)RNA on lipopolysaccharide (LPS)-mediated HMGB1 release, HMGB1-mediated hyper permeability and leukocyte migration were explored in vitro and in vivo. Furthermore, the effect of post-injection with 
PBX3 siRNA was also investigated regarding cecal ligation and puncture (CLP)-induced HMGB1 release, production of IL-6 and mortality. Collectively, the present study investigated whether PBX3 3'UTR modulates HMGB1 expression via acting as a ceRNA during sepsis.

\section{Materials and methods}

Cell culture. HUVECs (CBP60340) and RAW264.7 (CBP420599) cells were purchased from the Cell Bank in Chinese Academy of Sciences of China (Shanghai, China) and cultured in Dulbecco's Modified Eagle's Medium (DMEM; Gibco; Thermo Fischer Scientific, Inc., Waltham, MA, USA) supplemented with $10 \%$ fetal bovine serum (FBS; Gibco; Thermo Fischer Scientific, Inc.), $80 \mathrm{U} / \mathrm{ml}$ penicillin, $0.08 \mathrm{mg} / \mathrm{ml}$ streptomycin and $2 \mathrm{mM}$ glutamine at $37^{\circ} \mathrm{C}$ in a humidified atmosphere containing $5 \% \mathrm{CO}_{2}$.

Animal model of sepsis. 60 Male C57BL/6 mice (8-weeks old; 20-25 g) were purchased from the Medical Center of Yangzhou University (Yangzhou, China). Mice were housed and fed at $27^{\circ} \mathrm{C}$, with a $12 / 12 \mathrm{~h}$ light/dark cycle and free access to food/water. Each group (Saline, LPS, LPS + PBX3 siRNA, LPS + PBX3 siRNA + HMGB1, CLP and HMGB1) had at least 8 mice. All animal experiments were performed with the approval of the Qingzhou Hospital Ethics Committee for Animal Experimentation (Qingzhou, China). The sepsis model was induced using CLP as described previously (13). Mice were anesthetized via inhalation with $2.5 \%$ isoflurane (Shanghai Yuyan Instruments, Shanghai, China). A midline abdominal incision was made, and the cecum was exteriorized, ligated distal to the ileocecal valve and subsequently punctured twice with a 21-gauge needle. A small amount of feces was subsequently extruded into the abdominal cavity and the abdominal wall and skin were sutured in layers with 3-0 silk. Sham-operated mice were treated identically, with the exception that the cecum was neither ligated nor punctured. A total of $60 \mathrm{mg} / \mathrm{kg}$ in $100 \mu 1 \mathrm{PBX} 3$ siRNA (Biomics Biotechnologies Co., Ltd., China) was injected in the tail vein of mice during the sepsis.

ELISA. HUVECs or RAW264.7 cells with different treatments (saline, LPS + saline, LPS + PBX3 siRNA, HMGB1, HMGB1 + Lenti-PBX3-shRNA, LPS, LPS + PBX3 siRNA, LPS + PBX3 siRNA + HMGB1) were seeded at a density of $50 \times 10^{4} /$ well on 6 -well plates for $48 \mathrm{~h}$ at $37^{\circ} \mathrm{C}$. Subsequently, supernatants were collected and the concentrations of HMGB1, tumor necrosis factor (TNF)- $\alpha$ (cat. no. MTA00B, R\&D systems, Inc., Minneapolis, USA), interleukin (IL)-1 $\beta$ (cat. no. EK0394, Boster Biological Technology, Pleasanton, CA, USA) and IL-6 (cat. no. EK0411, Boster Biological Technology) were measured in mice blood, using ELISA kits according to the manufacturer's protocol. Furthermore, ELISA was used to measure the HMGB1 and IL-6 concentrations in septic mice. ELISA kits were purchased from R\&D Systems, Inc. (Minneapolis, MN, USA) and used according to the manufacturer's protocol.

Lentivirus vector construction. The pLKO.1-puro-PBX3, pLKO.1-puro-HMGB1 and pLKO.1-puro-Dicer1 plasmids were constructed by annealing at $37^{\circ} \mathrm{C}$ for $1 \mathrm{~h}$, forward and reverse oligonucleotides to each other. The resulting double-stranded DNA molecule was subsequently inserted into the AgeI/EcoRI sites of the pLKO.1-puro vector (Plasmid cat. no. 8453, Addgene Inc., Cambridge, MA, USA) and the following groups were established: Lenti-PBX3-shRNA, Lenti-HMGB1-shRNA and Lenti-Dicerl-shRNA. The empty pLKO.1-puro vector was used as a control. Fragments of PBX3 3'UTR or coding sequences were inserted into pLVX-IRES-ZsGreen1 (VT1460, Youbio; www.youbio.cn/, Changsha, China) and grouped in to the Lenti-PBX3-3'UTR and Lenti-PBX3-CDS, respectively. The packaging process of lentivirus and screening process of stable expression cell lines were performed as described previously (15). The primer sequences are presented in Table I.

Reverse transcription-quantitative polymerase chain reaction $(R T-q P C R)$. Total RNA was isolated from HUVECs using TRIzol reagent (Invitrogen; Thermo Fisher Scientific, Inc., Waltham, MA, USA) according to the manufacturer's protocol. Subsequently, $2 \mu \mathrm{g}$ RNA was reverse transcribed into cDNA using a Transcriptor cDNA Synthesis kit (Roche Diagnostics, Basel, Switzerland) according to the manufacturer's protocol. To detect the mRNA expression levels, the annealing temperature was $58^{\circ} \mathrm{C}$ and 35 cycles of RT-qPCR was performed in triplicate using a SYBR Green PCR Master Mix (Thermo Fisher Scientific, Inc.). The relative expression levels were calculated by comparing $\mathrm{Cq}$ values of the samples with those of the reference gene, $\beta$-actin (16). Primer sequences are presented in Table I.

Western blot analysis. Western blot analysis was performed following protein extraction from HUVECs as described previously (17). Antibodies against PBX3 (cat. no. ab56239, 1:1,000 at $4^{\circ} \mathrm{C}$ overnight) and HMGB1 (cat. no. ab79823, 1:5,000 at $4^{\circ} \mathrm{C}$ overnight) were purchased from Abcam (Cambridge, UK) and $\beta$-actin was purchased from OriGene Technologies, Inc. (Beijing, China). Blots were washed and incubated with a hydrogen peroxidase-conjugated secondary antibody (ab6728 or ab6721, Abcam, 1:5,000 for $1 \mathrm{~h}$ at room temperature), and chemiluminescence was detected using an enhanced chemiluminescence kit (Thermo Fisher Scientific, Inc.) followed by exposure with a Tanon 5200 Multi Chemiluminescent System.

Luciferase reporter assay. Fragments of the HMGB1 3'UTR and PBX3 3'UTR were cloned into pMiR-Report (VT1399, Youbia, Changsha, China) multiple cloning sites. The obtained vectors were defined as Luc-HMGB1-3'UTR and Luc-PBX3-3'UTR, respectively. The primer sequences are presented in Table I. HUVECs were co-transfected with Luc-HMGB1-3'UTR or Luc-PBX3-3'UTR and $\beta$-gal control plasmid (E1081, Promega Corporation) using Lipofectamine 2000 (Invitrogen; Thermo Fisher Scientific, Inc.) along with miRNA mimics miR-129, miR-142, miR-181, miR-200bc, miR-144-3p or miR-101-3p (Shanghai GenePhama Co., Ltd., Shanghai, China) or Lenti-PBX3-3'UTR. Following $72 \mathrm{~h}$ of transfection, cells were collected, and the luciferase activity was measured using a Glomax 96 luminometer (Promega Corporation, Madison, WI, USA). The transfection efficiency was normalized to $\beta$-gal activity. 
Table I. Primer sequences used in the present study.

\begin{tabular}{|c|c|c|}
\hline Gene & Direction & Sequence $\left(5^{\prime}-3^{\prime}\right)$ \\
\hline HMGB1 RT-qPCR & Forward & СТTССТCTTCTGCTCTGAGTATCG \\
\hline HMGB1 RT-qPCR & Reverse & СТTССТTСТTTTTCTTGСТTTTTT \\
\hline PBX3 RT-qPCR & Forward & CTGATAGAGGTGAAAACGAGATTG \\
\hline PBX3 RT-qPCR & Reverse & TTTGTATGAGTTCGCATGAGGTAA \\
\hline$\beta$-actin RT-qPCR & Forward & ATCCACGAAACTACCTTCAACTCC \\
\hline$\beta$-actin RT-qPCR & Reverse & GATCTTGATCTTCATTGTGCTGGG \\
\hline Lenti-PBX3-3'UTR lentivirus construction & Forward & GGACTAGTTGGCCTTGGAGGAAATTCACTGTAC \\
\hline Lenti-PBX3-3'UTR lentivirus construction & Reverse & GCTCTAGA AATCATGAAAGCAAAAAGTTTATTC \\
\hline Lenti-PBX3-CDS lentivirus construction & Forward & GGACTAGTATGGACGATCAATCCAGGATGCTGC \\
\hline Lenti-PBX3-CDS lentivirus construction & Reverse & GCTCTAGAGCAGCATCCTGGATTGATCGTCCAT \\
\hline Lenti-Dicer1-shRNA lentivirus construction & Forward & $\begin{array}{l}\text { CCGGAAGGCTTACCTTCTCCAGGCTCTCGAGAGC } \\
\text { CTGGAGAAGGTAAGCCTTTTTTTG }\end{array}$ \\
\hline Lenti-Dicer1-shRNA lentivirus construction & Reverse & $\begin{array}{l}\text { AATTCAAAAAAAGGCTTACCTTCTCCAGGCTCTC } \\
\text { GAGAGCCTGGAGAAGGTAAGCCTT }\end{array}$ \\
\hline Lenti-PBX3-shRNA lentivirus construction & Forward & $\begin{array}{l}\text { CCGGGGCGCAAGCAAAGAAACATCTCGAGATGTT } \\
\text { TCTTTGCTTGCGCCTTTTTG }\end{array}$ \\
\hline Lenti-PBX3-shRNA lentivirus construction & Reverse & $\begin{array}{l}\text { AATTCAAAAAGGCGCAAGCAAAGAAACATCTCG } \\
\text { AGATGTTTCTTTGCTTGCGCC }\end{array}$ \\
\hline Lenti-HMGB1-shRNA lentivirus construction & Forward & $\begin{array}{l}\text { CCGGGCAGCTTATACGAAATAATCTCGAGATTATT } \\
\text { TCGTATAAGCTGCTTTTTG }\end{array}$ \\
\hline Lenti-HMGB1-shRNA lentivirus construction & Reverse & $\begin{array}{l}\text { AATTCAAAAAGCAGCTTATACGAAATAATCTCGA } \\
\text { GATTATTTCGTATAAGCTGC }\end{array}$ \\
\hline Luc-HMGB1-3'UTR luciferase assay & Forward & GGACTAGTGTTGGTTCTAGCGCAGTTTTTTTTT \\
\hline Luc-HMGB1-3'UTR luciferase assay & Reverse & CССAAGCTTTCACTTTCTGGCTTTTATCTACTTT \\
\hline Luc-PBX3-3'UTR luciferase assay & Forward & GGACTAGTGGCCTTGGAGGAAATTCACTGTAC \\
\hline Luc-PBX3-3'UTR luciferase assay & Reverse & CCCAAGCTTATCATGAAAGCAAAAAGTTTATTC \\
\hline
\end{tabular}

PBX3, pre-B cell leukemia transcription factor 3; HMGB1, high mobility group box 1; shRNA, short hairpin RNA; UTR, untranslated region; CDS, coding sequence.

Permeability assay in vitro. HUVECs $\left(8 \times 10^{4} /\right.$ well) were plated in Transwell chambers with matrigel (EMD Millipore, Billerica, MA, USA) for $6 \mathrm{~h}$ at $37^{\circ} \mathrm{C}$ and then treated with HGMB1 $(1 \mu \mathrm{g} / \mathrm{ml})$ for $16 \mathrm{~h}$ followed by infection with Lenti-PBX3-shRNA for $24 \mathrm{~h}$ at $37^{\circ} \mathrm{C}$. Transwell inserts were subsequently washed with phosphate buffered saline, followed by the addition of $0.5 \mathrm{ml}$ Evans blue dye $(0.67 \mathrm{mg} / \mathrm{ml})$ diluted in DMEM medium containing $5 \%$ bovine serum albumin (BSA). A total of $800 \mu 1$ fresh DMEM medium with $20 \%$ FBS was added to the lower chamber, and the medium in the upper chamber was replaced with Evans blue/BSA. Following incubation for $10 \mathrm{~min}$ at $37^{\circ} \mathrm{C}$, the optical density was measured at $650 \mathrm{~nm}$ in the lower chamber.

In vivo permeability and leukocyte migration assays. CLP-operated or control mice were pretreated with HMGB1 (2 $\mu \mathrm{g}$ /mouse) intravenously for $16 \mathrm{~h}$ prior to receiving an intravenous injection of PBX3 siRNA (10 nM). A total of $48 \mathrm{~h}$ later, $1 \%$ Evans blue dye solution in normal saline was injected intravenously into the tail of each mouse. A total of $30 \mathrm{~min}$ later, the mice were sacrificed and the peritoneal exudates were collected once they were washed with $5 \mathrm{ml}$ normal saline and centrifuged at $200 \mathrm{x} \mathrm{g}$ for $10 \mathrm{~min}$ at $4^{\circ} \mathrm{C}$. The absorbance of the supernatant was read at $650 \mathrm{~nm}$. The vascular permeability was expressed according to the amount of dye ( $\mu \mathrm{g} /$ mouse). For assessing the leukocyte and neutrophil migration, the same method was performed prior to washing the peritoneal cavities with $5 \mathrm{ml}$ of normal saline. A total of $20 \mu \mathrm{l}$ peritoneal fluid was mixed with $0.38 \mathrm{ml} 0.01 \%$ crystal violet in $3 \%$ acetic acid at $37^{\circ} \mathrm{C}$ for $30 \mathrm{~min}$, and the leukocyte number was counted under a light microscope (magnification, x200).

Statistical analysis. All data were obtained from at least three independent experiments and expressed as the mean + standard deviation. Statistical analyses between two groups were performed using Student's t-test, and one-way analysis of variance was used for multiple-group comparisons, with Bonferroni-Holm or Tukey post hoc tests. $\mathrm{P}<0.05$ was considered to indicate a statistically significant difference.

\section{Results}

Enforced expression of PBX3 3'UTR promotes HMGB1 expression in HUVECs. Bioinformatics methods (miRNA.org 

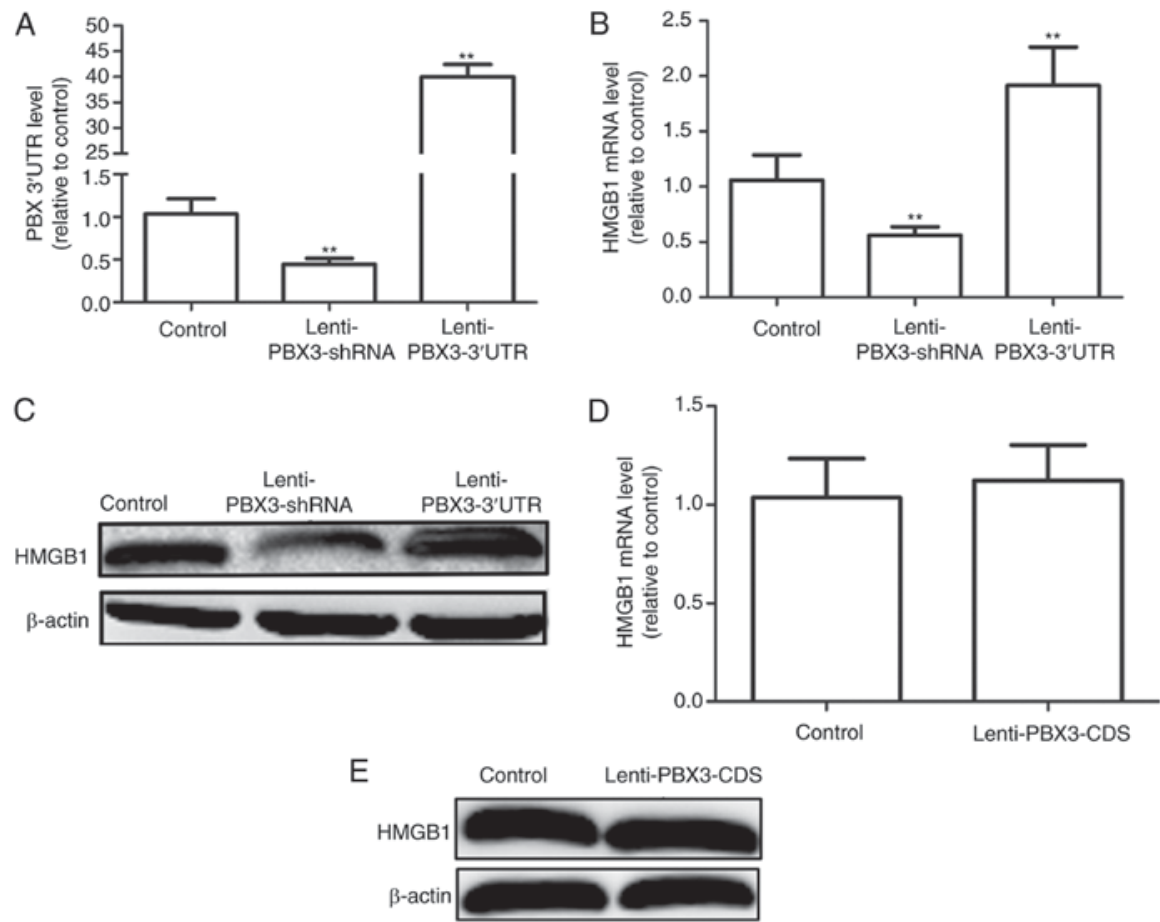

Figure 1.Ectopic expression of PBX3 3'UTR promoted HMGB1 expression in HUVECs. (A) Infection efficiency of Lenti-PBX3 3'UTR and Lenti-PBX3-shRNA was measured in HUVECs using RT-qPCR. (B) RT-qPCR and (C) western blotting was applied to examine the mRNA and protein expression levels of PBX3 in HUVECs with PBX3 3'UTR overexpression or knockdown. HMGB1 (D) mRNA and (E) protein expression levels were detected in HUVECs infected with Lenti-PBX3-CDS using RT-qPCR and western blotting. Data are presented as the mean + standard deviation. ${ }^{* *} \mathrm{P}<0.01 \mathrm{vs}$. Control. RT-qPCR, reverse transcription-quantitative polymerase chain reaction; shRNA, short hairpin RNA; UTR, untranslated region; CDS, coding sequence; PBX3, pre-B cell leukemia transcription factor 3; HMGB1, high mobility group box 1; HUVECs, human umbilical vein endothelial cells.

and targetscan.org) were used to predict the potential miRNA binding sites for PBX3 3'UTR and HMGB1 3'UTR. Results indicated that PBX3 3'UTR and HMGB1 3'UTR shared six common miRNA binding sites. Subsequently, it was determined whether PBX3 3'UTR may promote HMGB1 expression in HUVECs. Infection with Lenti-PBX3-3'UTR or Lenti-PBX3-shRNA significantly increased and decreased PBX3 3'UTR expression levels, respectively compared with the control $(\mathrm{P}<0.01$; Fig. 1A). Furthermore, overexpression of PBX3 3'UTR significantly increased HMGB1 mRNA expression levels, whereas downregulation of PBX3 exerted the opposite effects when compared with the control $(\mathrm{P}<0.01$; Fig. 1B). Similar effects were observed at the protein level (Fig. 1C). However, overexpression of the PBX3 CDS did not significantly alter the mRNA or protein expression levels of HMGB1 (Fig. 1D and E, respectively). Collectively, these results demonstrate that the PBX3 3'UTR, but not the PBX3 CDS, may positively modulate HMGB1 expression in HUVECs.

PBX3 3'UTR promotes HMGB1 expression by acting as a ceRNA for HMGB1. As PBX3 3'UTR and HMGB1 3'UTR shared six common miRNAs, it was hypothesized PBX3 3'UTR may operate as a ceRNA for HMGB1 in HUVECs. The present study explored whether the effect of PBX3 3'UTR on HMGB1 expression may be mediated by miRNAs. HUVECs were used with Dicer 1 knockdown, which is a ribonuclease that is critical for miRNA biogenesis, and its deficiency results in drastically reduced levels of mature miRNAs (3). Results demonstrated that knockdown of PBX3 significantly decreased
HMGB1 mRNA expression levels in Dicer 1-proficient cells $(\mathrm{P}<0.01)$, but not in that of isogenic Dicer 1 knockdown cells when compared with the control (Fig. 2A). Similar results were observed at the protein level (Fig. 2B). To further determine whether PBX3 3'UTR mediates its effect through its 3'UTR, co-expression of HMGB1 3'UTR-luciferase reporter (Luc-HMGB1-3'UTR) in Dicer 1-proficient and -deficient HUVECs with PBX3 knockdown was performed. Knockdown of PBX3 significantly decreased the activity of Luc-HMGB1-3'UTR; however, the opposite effect was observed in Dicer 1-knockdown cells when compared with the control $(\mathrm{P}<0.01$; Fig. $2 \mathrm{C})$. To further investigate this result, the Luc-HMGB1-3'UTR and Luc-PBX3-3'UTR activity in six predicted shared miRNAs (miR-129, miR-142, miR-181, miR-200bc, miR-144-3p, miR-101-3p) was measured using luciferase reporter assays. Results indicated that all six miRNAs significantly repressed Luc-HMGB1-3'UTR and Luc-PBX3-3'UTR activities compared with the control $(\mathrm{P}<0.01$; Fig. 2D and $\mathrm{E})$, which suggested that the crosstalk may be mediated by these shared miRNAs. Additionally, the absolute number of transcripts was determined using RT-qPCR. Plasmids carrying PBX3 3'UTR and HMGB1 3'UTR were used as standards. As indicated in Fig. 2F and G, PBX3 and HMGB1 were expressed at $1.14 \times 10^{10}$ and $8.27 \times 10^{10}$ in HUVECs, respectively. These results were consistent with a recent study, which suggested that ceRNA crosstalk was optimal when the transcript abundance of miRNAs and ceRNAs within a network was approximately equimolar (17). The present results support that PBX3 3'UTR may increase HMGB1 expression through its ceRNA activity. 


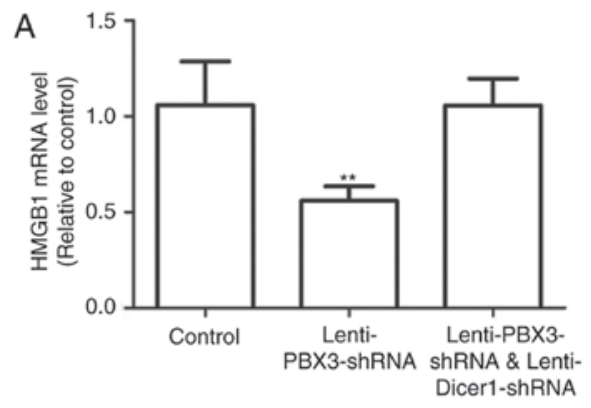

B
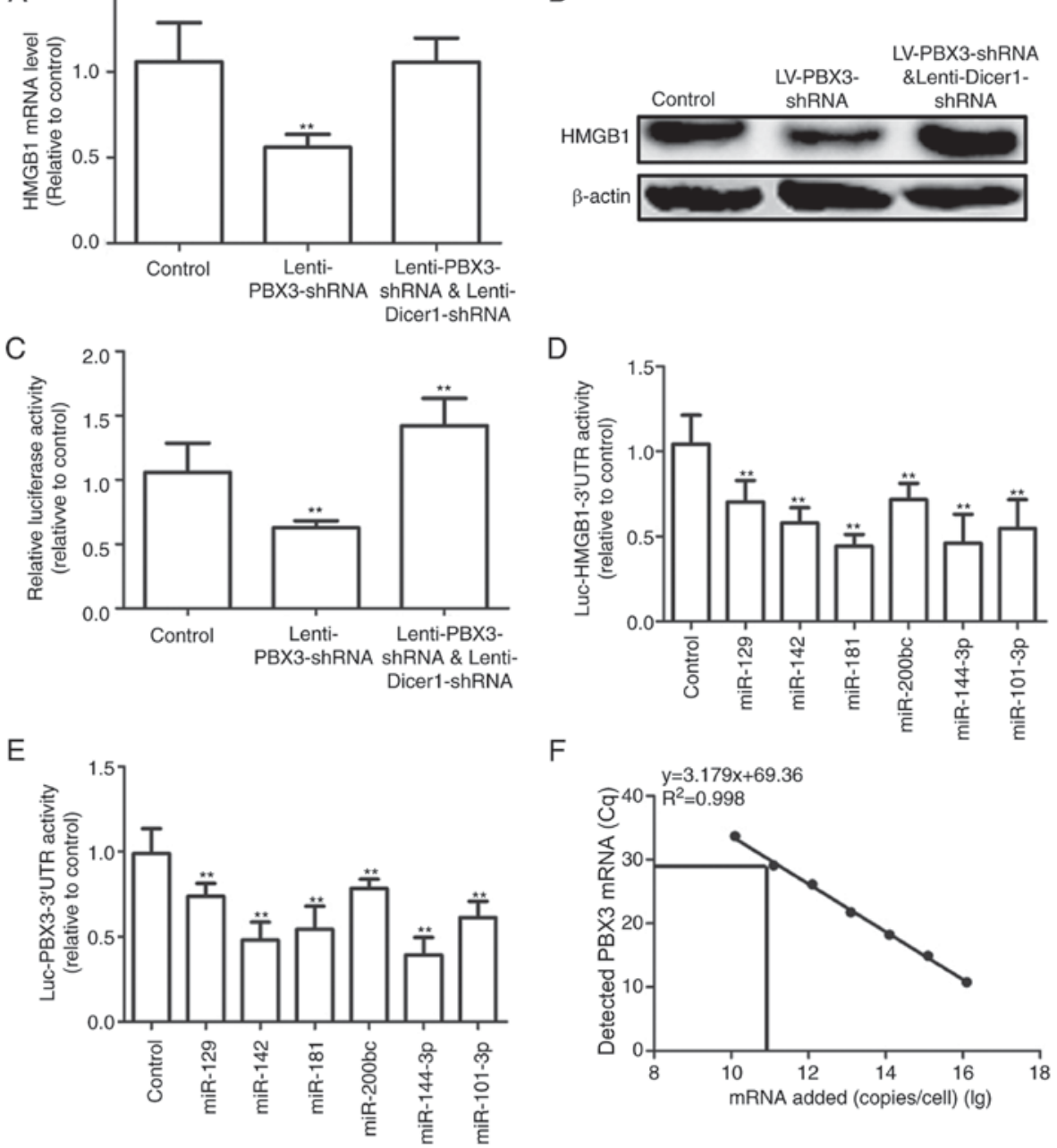

$\mathrm{F}$
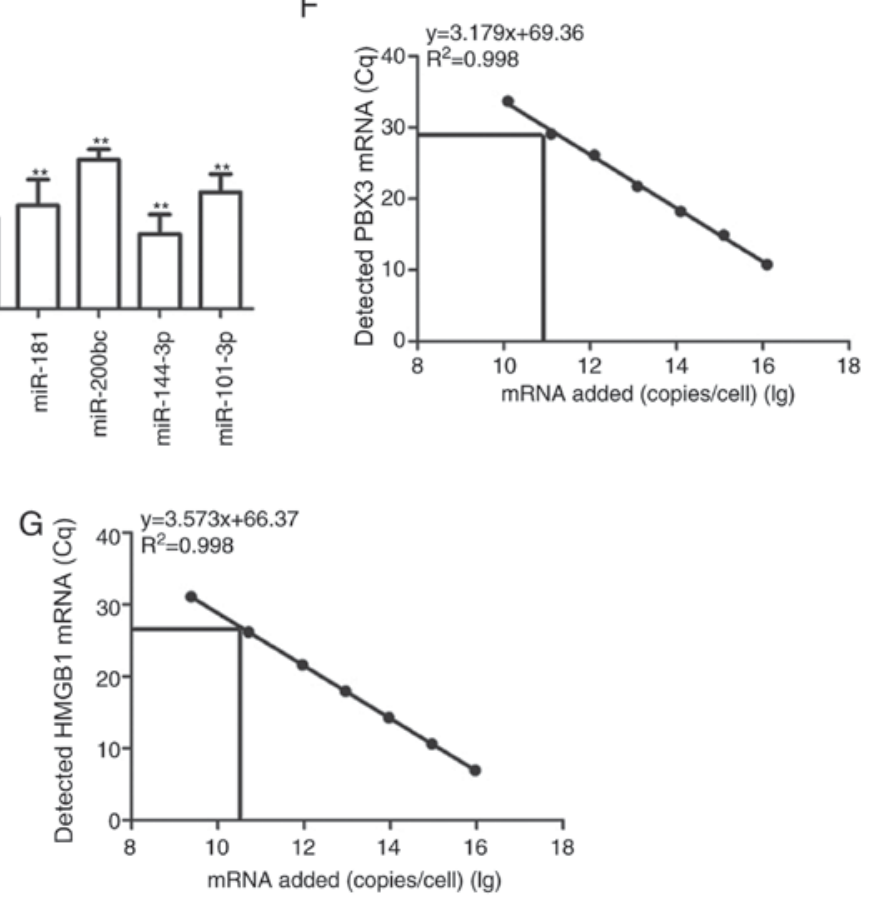

Figure 2. PBX3 3'UTR promoted HMGB1 expression by acting as a ceRNA for HMGB1. HMGB1 (A) mRNA and (B) protein expression levels were detected in HUVECs infected with Lenti-PBX3-shRNA, Lenti-PBX3-shRNAplus Lenti-Dicer1-shRNA and compared with the control. (C) Activity of Luc-HMGB1-3'UTR was measured in cells depicted in (A and B). Activities of (D) Luc-HMGB1-3'UTR and (E) Luc-PBX3-3'UTR were tested in HUVECs with miR-129, miR-142, miR-181, miR-200bc, miR-144-3p and miR-101-3p mimics. Absolute numbers of (F) PBX3 and (G) HMGB1 mRNAs were detected by reverse transcription-quantitative polymerase chain reaction combined with an internal standard curve. Values are presented as the mean + standard deviation $(n=3) .{ }^{* *} \mathrm{P}<0.01$ vs. control. ceRNA, competing endogenous RNA; shRNA, short hairpin RNA; UTR, untranslated region; PBX3, pre-B cell leukemia transcription factor 3; HMGB1, high mobility group box 1; HUVECs, human umbilical vein endothelial cells; miR, microRNA.

Pretreatment with PBX3 siRNA protects mice against lethal endotoxemia and inhibits the release of TNF- $\alpha$ and HMGB1. The present study evaluated whether PBX3 knockdown was able to exert a protective effect using a standard murine model of endotoxemia. Administration of PBX3 siRNA was followed $24 \mathrm{~h}$ later by the intraperitoneal administration of LPS (5 mg/kg). Injection with PBX3 siRNA significantly enhanced the percentage survival of mice from lethal endotoxemia $(10 / 15)$ compared with the LPS+saline-treated mice $(5 / 15 ; \mathrm{P}<0.01$;
Fig. 3A), which suggests that knockdown of PBX3 may not only delay the onset of LPS lethality. To further explore the protective mechanism of PBX3 knockdown against lethal endotoxemia, its effects were evaluated on the systemic accumulation of TNF- $\alpha$, which peak at $1 \mathrm{~h}$ following LPS administration (18), and HMGB1, whichislate-stage mediator of endotoxin lethality in mice (19). Pre-administration of PBX3 siRNA significantly decreased serum levels of TNF- $\alpha$ at 2 and $3 \mathrm{~h}$ (P<0.01; Fig. 3B) and HMGB1 at $24 \mathrm{~h}(\mathrm{P}<0.01$; Fig. 3C) following LPS treatment. 
A
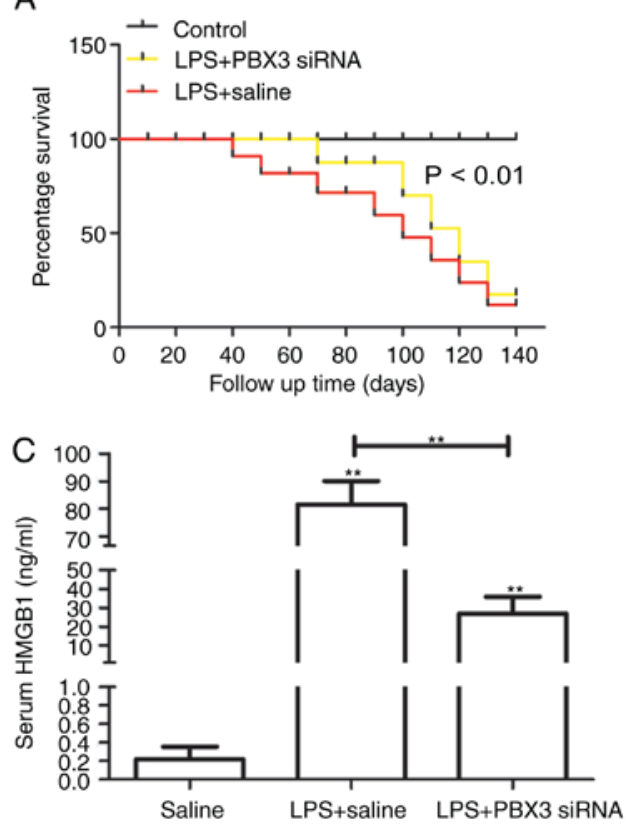
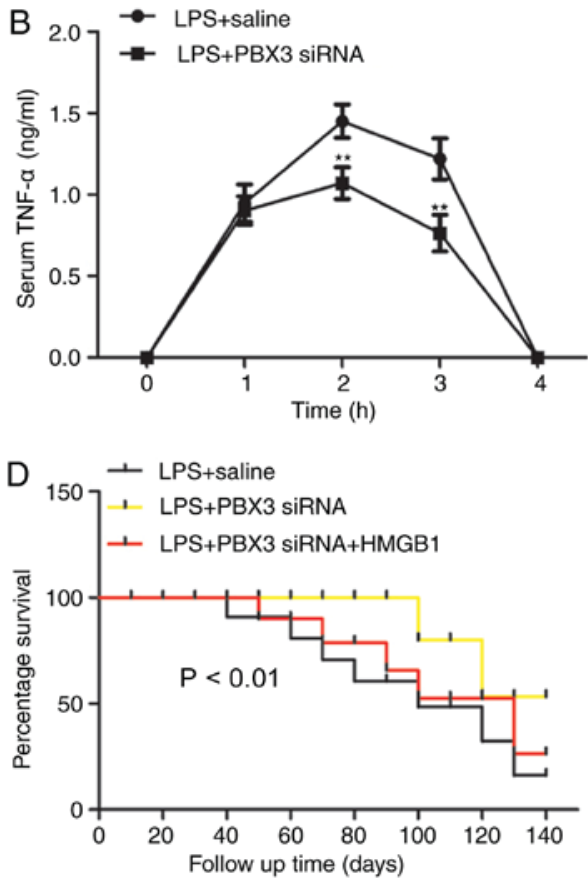

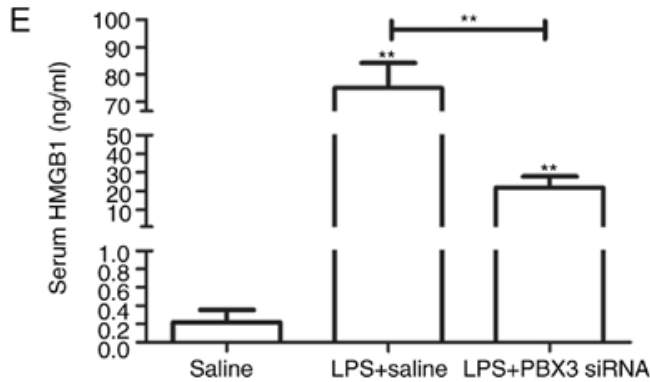

Figure 3. Pretreatment with PBX3 siRNA protected mice against lethal endotoxemia and inhibited the release of TNF- $\alpha$ and HMGB1. (A) Mice (n=15 per group) were injected with PBX3 siRNA, followed 30 min later by a lethal infusion of endotoxin (LPS; $5 \mathrm{mg} / \mathrm{kg}$ intraperitoneal) and followed with PBX3 siRNA injection every three days. The Kaplan-Meyer method was used to compare the differences in survival rates between groups. In parallel, serum levels of (B) TNF- $\alpha$ at 1-4 $\mathrm{h}$ and (C) HMGB1 at $24 \mathrm{~h}$ were measured by ELISA ( $\mathrm{n}=6$ mice per group). (D) Mice ( $\mathrm{n}=15$ per group) were injected with endotoxin (LPS, $5 \mathrm{mg} / \mathrm{kg}$ ), followed $30 \mathrm{~min}$ later by PBX3 siRNA injection every three days. The Kaplan-Meyer method was used to compare the differences in survival rates between groups. (E) Serum level of HMGB1 at $32 \mathrm{~h}$ was measured by ELISA ( $\mathrm{n}=6$ mice per group). Values are presented as the mean + standard deviation $(\mathrm{n}=3) .{ }^{* *} \mathrm{P}<0.01$ vs. $\mathrm{LPS}+$ saline for $(\mathrm{A}),{ }^{* *} \mathrm{P}<0.01$ vs. LPS $+\mathrm{PBX}$ siRNA for $(\mathrm{D}),{ }^{* *} \mathrm{P}<0.01$ vs. saline or as indicated. PBX3, pre-B cell leukemia transcription factor 3; HMGB1, high mobility group box 1; LPS, lipopolysaccharide; siRNA, small interfering RNA; TNF- $\alpha$, tumor necrosis factor- $\alpha$.

These findings demonstrate that PBX3 knockdown inhibited endotoxemia by downregulating the release of early and late cytokines. To investigate whether PBX3 knockdown may be delayed following LPS administration and still maintain its protective effects, PBX3 siRNA was administered $5 \mathrm{~h}$ after LPS. As indicated in Fig. 3D, this delayed treatment significantly enhanced the percentage survival against lethal endotoxemia compared with LPS+saline alone; however, this protective effect was reversed with HMGB1 injection via tail vein $(2 \mu \mathrm{g} / \mathrm{mouse}$; followed at $48 \mathrm{~h}$ after LPS). In addition, treatment of mice with PBX3 siRNA beginning at $3 \mathrm{~h}$ following LPS injection, which was defined as the onset of endotoxemia (20), significantly attenuated the systemic release of HMGB1 measured at $32 \mathrm{~h}$. $(\mathrm{P}<0.01$; Fig. 3E). These results indicate that delayed treatment with PBX3 siRNA significantly enhances protection, partly by inhibiting HMGB1 release.

Effects of PBX3 siRNA on LPS-, HMGB1- and CLP-mediated barrier disruption. Since PBX3 knockdown was indicated to attenuate the release of HMGB1, the effect of PBX3 knockdown on the barrier integrity of HUVECs was further determined. The barrier integrity was not significantly affected with PBX3 knockdown alone (Fig. 4A). As endothelial membrane barriers maybe disrupted by LPS induction (20), HUVECs were infected with Lenti-PBX3-shRNA for $24 \mathrm{~h}$ following LPS treatment (100 ng/ml). Results indicated that PBX3 knockdown significantly attenuated the LPS-mediated membrane disruption $(\mathrm{P}<0.01$; Fig. 4B). Furthermore, the barrier integrity may be perturbed by HMGB1 induction (Fig. 4D and E). Therefore, HUVECs were infected with Lenti-PBX3-shRNA for $24 \mathrm{~h}$ following HMGB1 protein addition $(1 \mu \mathrm{g} / \mathrm{ml})$. As indicated in Fig. 4C, infection with Lenti-PBX3-shRNA significantly inhibited HMGB1-mediated membrane disruption $(\mathrm{P}<0.01)$.

The effects of PBX3 siRNA on HMGB1- and CLP-mediated barrier disruption were investigated in vivo by examining HMGB1- or CLP-induced vascular permeability in mice. Treatment with PBX3 siRNA significantly decreased the level of HMGB1- and CLP-induced peritoneal leakage of 

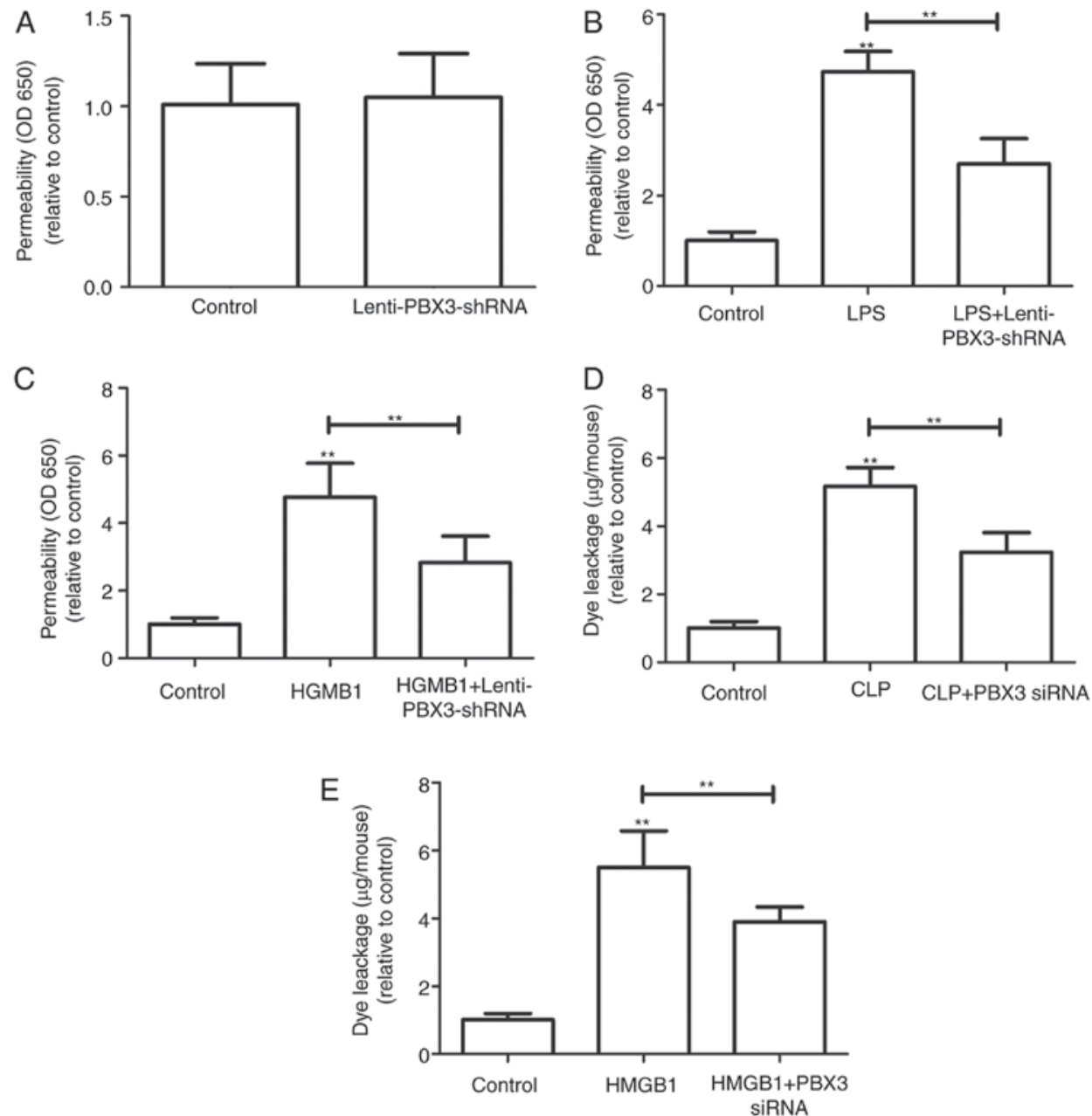

Figure 4. Effects of PBX3 siRNA on LPS-, HMGB1- and CLP-mediated barrier disruption. (A) HUVECs were infected with Lenti-PBX3-shRNA for 24 h. Permeability was monitored by measuring the flux of Evans blue-bound albumin across HUVECs. HUVECs were stimulated with (B) LPS (100 ng/ml for $4 \mathrm{~h}$ ) or (C) HMGB1 $(1 \mu \mathrm{g} / \mathrm{ml}$ for $16 \mathrm{~h})$ and then infected with Lenti-PBX3-shRNA for $24 \mathrm{~h}$. Permeability was monitored by measuring the flux of Evans blue-bound albumin across HUVECs. Effects of PBX3 siRNA injection intravenously on (D) CLP-induced (at $24 \mathrm{~h}$ after CLP) or (E) HMGB1-induced (2 $\mu \mathrm{g} / \mathrm{mouse}$, intravenously) vascular permeability in mice were examined by measuring the amount of Evans blue dye in peritoneal washings (expressed as $\mu \mathrm{g} / \mathrm{mouse}, \mathrm{n}=6$ ). Values were expressed as the mean + standard deviation $(n=3){ }^{* * *} \mathrm{P}<0.01$ vs. Control or as indicated. PBX3, pre-B cell leukemia transcription factor 3; HMGB1, high mobility group box 1; LPS, lipopolysaccharide; siRNA, small interfering RNA; shRNA, short hairpin RNA; CLP, cecal ligation and puncture; HUVECs, human umbilical vein endothelial cells; OD, optical density.

dye compared with HMGB1 or CLP groups alone $(\mathrm{P}<0.01$; Fig. 4D and E). These results demonstrate that knockdown of PBX3 may ameliorate HMGB1- or CLP-mediated endothelial disruption and maintain endothelial cell barrier integrity.

$P B X 3$ knockdown exerts protection during sepsis in a HMGB1-dependent manner. To demonstrate that the protective effects of PBX3 knockdown occurred via inhibition of HMGB1 release, the production of IL-1 $\beta$ was detected in HMGB1-activated HUVECs. The production of IL-1 $\beta$ was significantly increased following HMGB1 treatment compared with the control, and this was significantly attenuated by PBX3 knockdown $(\mathrm{P}<0.01$; Fig. 5A). However, following co-infection with Lenti-Dicer1-shRNA and Lenti-PBX3-shRNA, PBX3 knockdown did not significantly inhibit the effects of HMGB1 treatment on IL-1 $\beta$ release (Fig. 5B). Additionally, the effects of PBX3 knockdown on mice treated with HMGB1 protein following LPS insult and PBX3 siRNA treatment were explored. SerumIL-1 $\beta$ production in response to PBX knockdown was partially reversed (Fig. 5C). Notably, PBX3 knockdown also significantly inhibited HMGB1-induced IL-1 $\beta$ upregulation in RAW264.7 cells ( $\mathrm{P}<0.01$; Fig. 5D). Collectively, these findings suggest that PBX3 knockdown may have protective effects during sepsis in an HMGB1-dependent manner, with which Dicer 1 expression is associated.

PBX3 knockdown inhibits cytokine activity and confers protective effects during sepsis. To explore whether PBX3 knockdown inhibits the cytokine activity of HMGB1, the murine macrophage cell line RAW264.7 was treated with Lenti-PBX3-shRNA following HMGB1 treatment. Results suggested that HMGB1-induced TNF- $\alpha$ and IL- 6 release was significantly inhibited by $\mathrm{PBX} 3$ knockdown in these cells $(\mathrm{P}<0.01$; Fig. 6A and $\mathrm{B})$. It was subsequently assumed that PBX3 knockdown would reduce the production of IL-6 in the HMGB1- or CLP-induced sepsis mouse model. As expected, production of IL- 6 in HMGB1- or CLP-induced mice was significantly reduced by $\mathrm{PBX} 3$ siRNA treatment $(\mathrm{P}<0.01$; Fig. 6C and D). These findings suggest that PBX3 may be a potential therapeutic target for sepsis. 

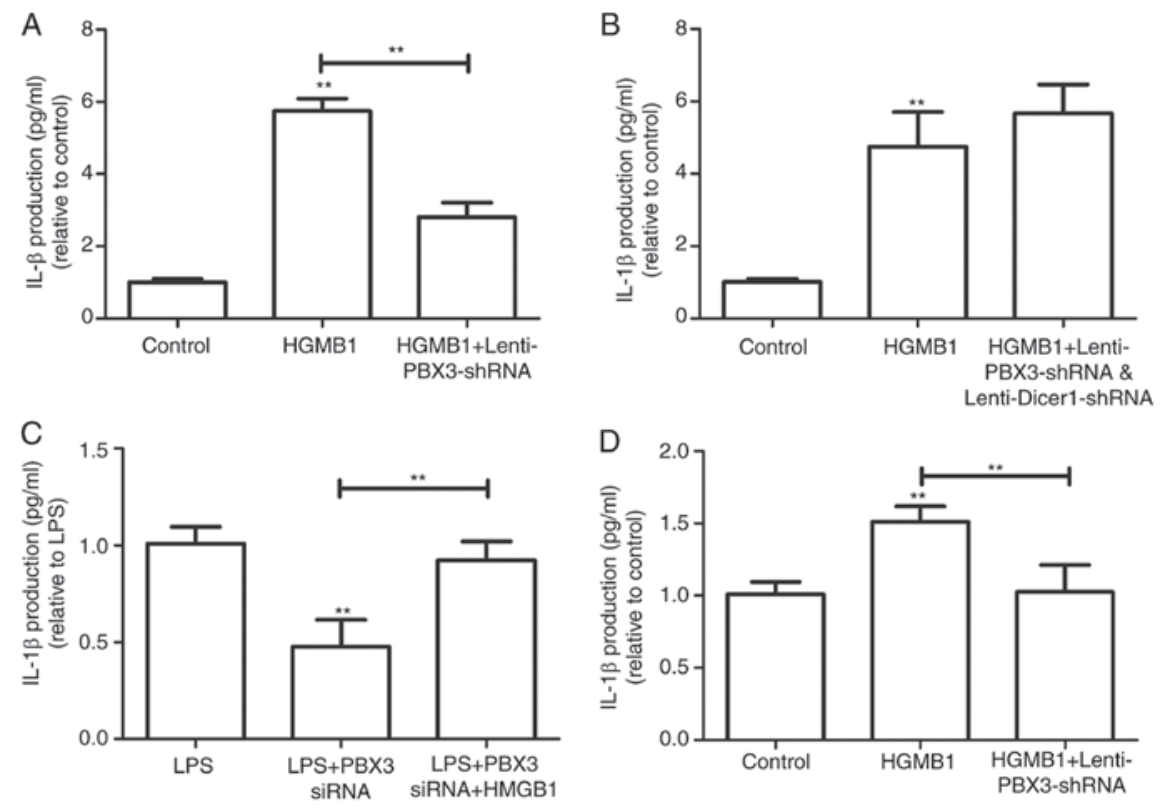

Figure 5. PBX3 knockdown exerted protection against sepsis in a HMGB1-dependent manner. (A) HUVECs were stimulated with HMGB1 (1 $\mu \mathrm{g} / \mathrm{ml})$ for $16 \mathrm{~h}$ followed by infection with Lenti-PBX3-shRNA. HMGB1-mediated expression levels of IL-1 $\beta$ in HUVECs were detected using ELISA. (B) HUVECs were stimulated with HMGB1 $(1 \mu \mathrm{g} / \mathrm{ml})$ for $16 \mathrm{~h}$ followed by infection with Lenti-PBX3-shRNA and Lenti-Dicer1-shRNA. HMGB1-mediated expression levels of IL-1 $\beta$ in HUVECs were detected using ELISA. (C) Mice ( $\mathrm{n}=6$ per group) were administered with endotoxin (LPS, $5 \mathrm{mg} / \mathrm{kg}$, intraperitoneally), followed 30 min later by PBX3 siRNA injection. Serum IL-1 $\beta$ levels were detected using ELISA. (D) IL-1 $\beta$ levels were measured in RAW264.7 cells with HMGB1 and Lenti-PBX3-shRNA infection. Values were expressed as the mean + standard deviation $(n=3)$. ${ }^{* *} \mathrm{P}<0.01$ vs. Control or as indicated. $\mathrm{PBX} 3$, pre-B cell leukemia transcription factor 3; HMGB1, high mobility group box 1; LPS, lipopolysaccharide; shRNA, short hairpin RNA; HUVECs, human umbilical vein endothelial cells; IL, interleukin.
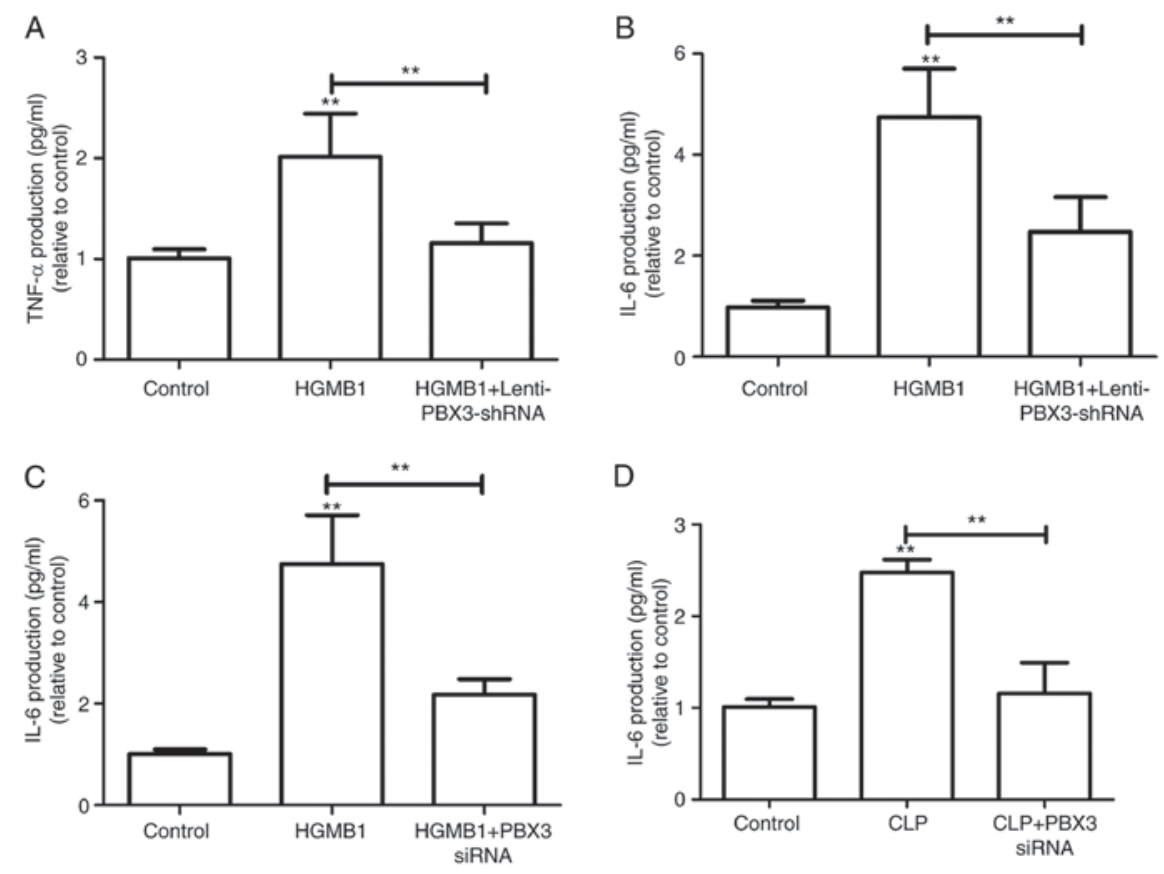

Figure 6. PBX3 knockdown inhibits the cytokine activity and confers protective effects during sepsis. (A) TNF- $\alpha$ and (B) IL-6 levels were measured in RAW264.7 cells with HMGB1 addition alone or plus Lenti-PBX3-shRNA infection. Effects of PBX3 siRNA injection intravenously on (C) HMGB1-induced ( $2 \mu \mathrm{g} /$ mouse, intravenously) or (D) CLP-induced (at $24 \mathrm{~h}$ after CLP) IL-6 secretion in mice was examined using ELISA (expressed $\mu \mathrm{g} / \mathrm{mouse}, \mathrm{n}=6$ ). Values were expressed as the mean + standard deviation $(n=3) .{ }^{* *} \mathrm{P}<0.01$ vs. Control or as indicated. PBX3, pre-B cell leukemia transcription factor 3; HMGB1, high mobility group box 1; LPS, lipopolysaccharide; shRNA, short hairpin RNA; IL, interleukin; CLP, cecal ligation and puncture.

\section{Discussion}

Thepresentstudyinvestigated whethertheHMGB1-associatedce RNA network exerts critical functions during sepsis, and whether perturbations in this network contribute to the delay of sepsis. The present study focused on PBX3 due to current understanding of its existence in humans and mice, and its deregulation in various types of cancer $(10,11)$. The present 
results established PBX3 as a potent molecule that may elicit sepsis. Notably, overexpression of the PBX3 CDS did not significantly influence HMGB1 expression; however, upregulation of the PBX3 3'UTR significantly increased HMGB1 expression in HUVECs in a $3^{\prime} \mathrm{UTR} \%$ and miRNA-dependent manner. While it is possible that PBX3 may elicit its effects through various mechanisms or signaling pathways, the present results provide evidence indicating that PBX3 may promote HMGB1 expression and sepsis via acting as ceRNA for HMGB1. Future investigations will focus on whether other additional ceRNA targets or non-ceRNA-related mechanisms maybe associated with the promotive effects of PBX3 3'UTR during sepsis, and whether other genes serve as ceRNAs for HMGB1.

Experimentally, the CLP model is considered as the most clinically relevant experimental model for human sepsis (21), thus this model was utilized in the present study. The present findings suggested that PBX3 knockdown attenuated LPS- and CLP-mediated HMGB1 release, ameliorated HMGB1-mediated endothelial disruption and maintained endothelial cell barrier integrity. Notably, the present results also indicated that PBX3 knockdown exerted protective effects on the CLP-induced production of IL-6 and septic mortality. Mechanistically, HMGB1 has been identified as a bio-marker of sepsis. However, the associated mechanisms modulating HMGB1 expression are not well established. The ceRNA network assigns functions to the non-coding parts of coding transcripts (3). Notably, as some genes are large, traditional cloning techniques may not achieve expression in plasmids (22). Therefore, the use of non-coding moieties, including 3'UTRs, which are relatively small in size, may partially overcome this difficulty. For example, a recent study indicated that pseudogene CYP4Z2P 3'UTR was used as a model to investigate the role of CYP4Z2P during breast cancer progression (22).

In the present study, HGMB1 was identified as a potential novel ceRNA target of PBX3 in HUVECs. To the best of our knowledge, the present study is the first to reveal the novel mechanism of PBX 3'UTR-mediated regulation for HGMB1 in HUVECs. In conclusion, by uncovering the mechanisms of PBX3 3'UTR-mediated regulation of HMGB1, the present findings not only provide insights into the modulation of HMGB1 expression during sepsis, but also provides a potent rationale for the development of HGMB1-based therapeutic intervention for sepsis.

\section{References}

1. Riedemann NC, Guo RF and Ward PA: Novel strategies for the treatment of sepsis. Nat Med 9: 517-524, 2003.

2. Phelps M, Coss C, Wang H and Cook M; Reproducibility Project; Cancer Biology; Reproducibility Project Cancer Biology: Registered report: Coding-independent regulation of the tumor suppressor PTEN by competing endogenous mRNAs. Elife 5: pii: e12470, 2016.

3. Li X, Zheng L, Zhang F, Hu J, Chou J, Liu Y, Xing Y and Xi T: STARD13-correlated ceRNA network inhibits EMT and metastasis of breast cancer. Oncotarget 7: 23197-23211, 2016.
4. Tay Y, Karreth FA and Pandolfi PP: Aberrant ceRNA activity drives lung cancer. Cell Res 24: 259-260, 2014.

5. Zhang $\mathrm{H}$, Wang $\mathrm{F}$ and $\mathrm{Hu} \mathrm{Y}$ : STARD13 promotes hepatocellular carcinoma apoptosis by acting as a ceRNA for Fas. Biotechnol Lett 39: 207-217, 2017.

6. Wang Y and Lin G: TP53INP1 3'-UTR functions as a ceRNA in repressing the metastasis of glioma cells by regulating miRNA activity. Biotechnol Lett 38: 1699-1707, 2016.

7. Valdés-Ferrer SI, Papoin J, Dancho ME, Olofsson P, Li J, Lipton JM, Avancena P, Yang H, Zou YR, Chavan SS, et al: HMGB1 mediates anemia of inflammation in murine sepsis survivors. Mol Med: Dec 29, 2015 (Epub ahead of print).

8. Yang M, Cao L, Xie M, Yu Y, Kang R, Yang L, Zhao M and Tang D: Chloroquine inhibits HMGB1 inflammatory signaling and protects mice from lethal sepsis. Biochem Pharmacol 86: 410-418, 2013.

9. Zhou W, Wang J, Li Z, Li J and Sang M: MicroRNA-2055b inhibits HMGB1 expression in LPS-induced sepsis. Int J Mol Med 38: 312-318, 2016.

10. Wang S, Li C, Wang W and Xing C: PBX3 promotes gastric cancer invasion and metastasis by inducing epithelial-mesenchymal transition. Oncol Lett 12: 3485-3491, 2016.

11. Ramberg H, Grytli HH, Nygård S, Wang W, Ögren O, Zhao S, Løvf M, Katz B, Skotheim RI, Bjartell A, et al: PBX3 is a putative biomarker of aggressive prostate cancer. Int J Cancer 139: 1810-1820, 2016.

12. Han HB, Gu J, Ji DB, Li ZW, Zhang Y, Zhao W, Wang LM and Zhang ZQ: PBX3 promotes migration and invasion of colorectal cancer cells via activation of MAPK/ERK signaling pathway. World J Gastroenterol 20: 18260-18270, 2014.

13. Denzler R, Agarwal V, Stefano J, Bartel DP and Stoffel M: Assessing the ceRNA hypothesis with quantitative measurements of miRNA and target abundance. Mol Cell 54: 766-776, 2014.

14. Zhang Z, Huang A, Zhang A and Zhou C: HuR promotes breast cancer cell proliferation and survival via binding to CDK3 mRNA. Biomed Pharmacother 91: 788-795, 2017.

15. Xu CZ, Jiang C, Wu Q, Liu L, Yan X and Shi R: A feed-forward regulatory loop between HuR and the long noncoding RNA HOTAIR promotes head and neck squamous cell carcinoma progression and metastasis. Cell Physiol Biochem 40: 1039-1051, 2016.

16. Livak KJ and Schmittgen TD: Analysis of relative gene expression data using real-time quantitative PCR and the 2(-Delta Delta C(T)) method. Methods 25: 402-408, 2001.

17. Zheng L, Li X, Meng X, Chou J, Hu J, Zhang F, Zhang Z, Xing Y, Liu Y and Xi T: Competing endogenous RNA networks of CYP4Z1 and pseudogene CYP4Z2P confer tamoxifen resistance in breast cancer. Mol Cell Endocrinol 427: 133-142, 2016.

18. Tracey KJ, Beutler B, Lowry SF, Merryweather J, Wolpe S, Milsark IW, Hariri RJ, Fahey TJ III, Zentella A, Albert JD, et al: Shock and tissue injury induced by recombinant human cachectin. Science 234: 470-474, 1986.

19. Wang H, Bloom O, Zhang M, Vishnubhakat JM, Ombrellino M, Che J, Frazier A, Yang H, Ivanova S, Borovikova L, et al: HMG-1 as a late mediator of endotoxin lethality in mice. Science 285 : 248-251, 1999.

20. Ishikawa M, Yamashita H, Oka N, Ueda T, Kohama K, Nakao A and Kotani JL: Antithrombin III improved neutrophil extracellular traps in lung after the onset of endotoxemia. J Surg Res 208: 140-150, 2017.

21. Li W, Zhu S, Zhang Y, Li J, Sama AE, Wang P and Wang H: Use of animal model of sepsis to evaluate novel herbal therapies. J Vis Exp: pii: 3926, 2012.

22. Zheng L, Li X, Gu Y, Ma Y and Xi T: Pseudogene CYP4Z2P 3'UTR promotes angiogenesis in breast cancer. Biochem Biophys Res Commun 453: 545-551, 2014.

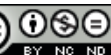

This work is licensed under a Creative Commons Attribution-NonCommercial-NoDerivatives 4.0 International (CC BY-NC-ND 4.0) License. 